\title{
Iron-free detector magnet options for the future circular collider
}

\author{
Matthias Mentink, Alexey Dudarev, Helder Filipe Pais Da Silva, Gabriella Rolando, Benoit Cure, \\ Andrea Gaddi, Vyacheslav Klyukhin, Hubert Gerwig, Udo Wagner, and Herman ten Kate \\ CERN, CH-1211 Geneva 23, Switzerland
}

(Received 9 July 2016; published 16 November 2016)

\begin{abstract}
In this paper, several iron-free solenoid-based designs of a detector magnet for the future circular collider for hadron-hadron collisions (FCC-hh) are presented. The detector magnet designs for FCC-hh aim to provide bending power for particles over a wide pseudorapidity range $(0 \leq|\eta| \leq 4)$. To achieve this goal, the main solenoidal detector magnet is combined with a forward magnet system, such as the previously presented force-and-torque-neutral dipole. Here, a solenoid-based alternative, the so-called balanced forward solenoid, is presented which comprises a larger inner solenoid for providing bending power to particles at $|\eta| \geq 2.5$, in combination with a smaller balancing coil for ensuring that the net force and torque on each individual coil is minimized. The balanced forward solenoid is compared to the force-and-torqueneutral dipole and advantages and disadvantages are discussed. In addition, several conceptual solenoidbased detector magnet designs are shown, and quantitatively compared. The main difference between these designs is the amount of stray field reduction that is achieved. The main conclusion is that shielding coils can be used to dramatically reduce the stray field, but that this comes at the cost of increased complexity, magnet volume, and magnet weight and reduced affordability.
\end{abstract}

DOI: 10.1103/PhysRevAccelBeams.19.111001

\section{INTRODUCTION}

The future circular collider for hadron-hadron physics (FCC-hh) is a conceptual design study that aims to develop a successor to the Large Hadron Collider (LHC) at CERN [1]. Compared to the LHC, particles are to collide at a seven times higher energy, which means that more powerful detector magnets are needed to provide the required bending power for identifying the collision products.

Previously, two conceptual designs were published. First, the "Twin Solenoid and Force-and-Torque-neutral dipoles" design (Figs. 1,2,3), which comprises two concentric solenoids for providing bending power for low-pseudo-rapidity particles and forward dipoles for high-pseudorapidity particles [2]. And second, the "Minimum yoke solenoid"-design, a design that is similar to the compact muon solenoid [3], comprising a superconducting solenoid and an iron yoke [4]. In this paper, a number of alternative conceptual detector magnet designs are presented, all of which comprise superconducting solenoids without iron.

Special attention is on the "balanced forward solenoid" (BFS), a new design and potential alternative to the forward dipole. Similarly to the earlier presented forward dipole, this design features a special geometry to minimize the

Published by the American Physical Society under the terms of the Creative Commons Attribution 4.0 International license. Further distribution of this work must maintain attribution to the author(s) and the published article's title, journal citation, and DOI. net force and torque on each individual coil during operation (see Sec. II). The advantages and disadvantages versus an extended solenoid and a solenoid in combination with forward dipoles are discussed in Secs. III and IV, respectively. A field integral comparison is presented in Sec. V.

In addition, various solenoid-based variants are presented in Sec. VI and compared in terms of stray field (Sec. VII) and general magnet properties (Sec. VIII).

Two major points are emphasized here. First, the advantages of utilizing a forward solenoid instead of a forward dipole are major. Second, it is demonstrated that the stray field can be strongly reduced and the field integral for muon tracking strongly enhanced through the use of shielding coils, but the use of these shielding coils comes at significant additional cost in terms of complexity, size, and weight.

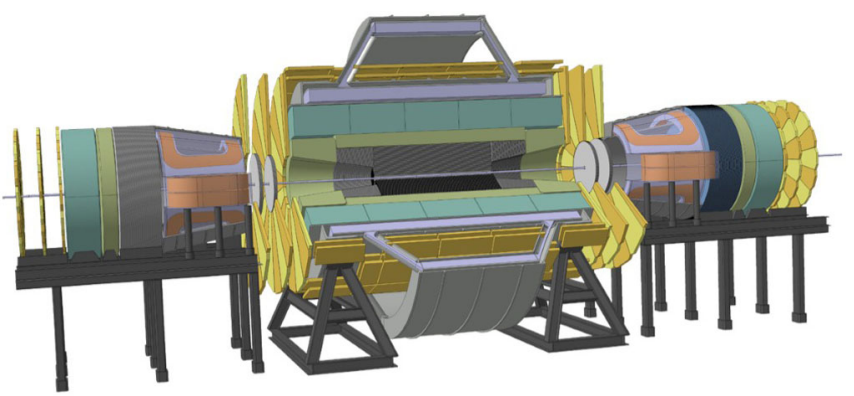

FIG. 1. Twin solenoid detector with forward dipoles detector layout. Also shown are the hadron calorimeters in dark green, the electromagnetic calorimeters in light green, the trackers in grey, and the muon chambers in yellow. 


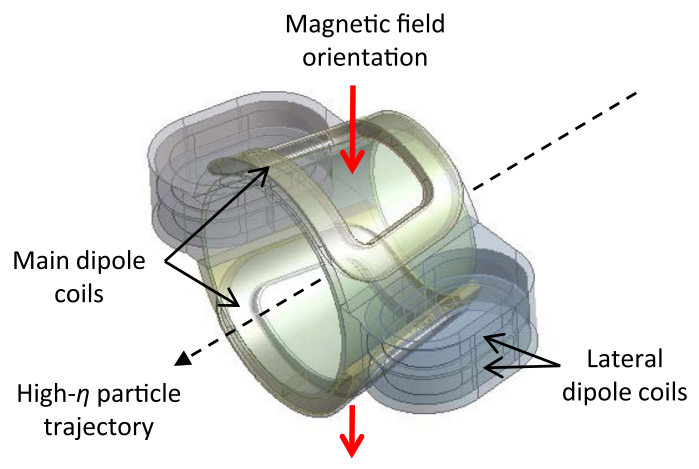

FIG. 2. Three-dimensional representation of the cold mass of the force-and-torque-neutral dipole. The main dipole coils contribute most of the useful field integral for particle tracking and the lateral coils return the flux generated by the main coil, thus reducing the stray field and canceling the net force and torque on the cold mass. See [2] for more details.

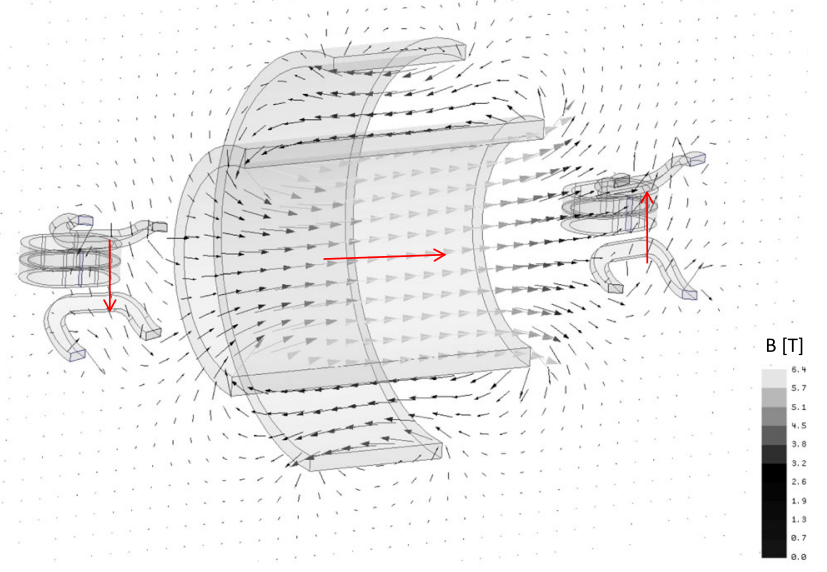

FIG. 3. Magnetic field map of forward dipoles in combination with twin solenoid. The dipoles are arranged in an up-down configuration. The red arrows indicate the solenoidal and dipole field orientations. It is clear that the dipoles are located very close to the twin solenoid, so that the stray field from the twin solenoid on the dipole conductor is as high at $1 \mathrm{~T}$.

\section{BALANCED FORWARD SOLENOID}

The balanced forward solenoid is an alternative to the forward dipole as discussed in [2] which provides a solenoidal magnetic field in line with the twin solenoid. Placing a magnet in close vicinity to another magnet presents a challenge in terms of forces and torques. For instance, removing the lateral dipole coils from the forceand-torque-neutral dipoles would result in a net torque of $170 \mathrm{MNm}$ and an off-axis force of $23 \mathrm{MN}$. Similarly, without special measures to compensate for the force, replacing the forward dipole with a solenoid of similar dimensions would result in a net axial force of several hundred MN with respect to the main solenoid. This force can be either attractive or repulsive, depending on whether current in the main and forward solenoids flows in the same or opposite direction, respectively. The cold masses of superconducting magnets are held in place by support structure and some net forces are acceptable, but accommodating a force of such high magnitude seems impractical.

To circumvent the forces problem, the balanced forward solenoid concept comprises an inner forward solenoid and a outer forward "balancing" solenoid (Figs. 4 and 5). Whereas in the inner forward solenoid and the inner main solenoid the current flows in the same direction (leading to a net attractive force), the current in the outer forward solenoid flows in opposite direction, resulting in a repulsive force with respect to both the inner main solenoid and the inner forward solenoid. With a properly balanced geometry, the attractive and repulsive forces on each coil counter each other exactly, so that the net force and torque on each of the coils are zero. The net axial force on each coil depends on the total amount of ampere-turns, the radius of the coil, and the radial magnetic field component generated by the other coils. As the balancing solenoid sees a large radial magnetic field component from the twin solenoid and has a larger average radius than the inner forward solenoid, it is comparatively compact (Fig. 5).

The inner forward solenoid is made conical and sufficiently large for particles at pseudorapidity $|\eta| \geq 2.5$ to pass through the free bore. Using a conical coil is not strictly necessary; the same purpose may be achieved with a cylindrical coil, but to allow particles at $|\eta|=2.5$ to pass through the free bore, the average bore radius, stored energy, and cold mass would be obviously larger.

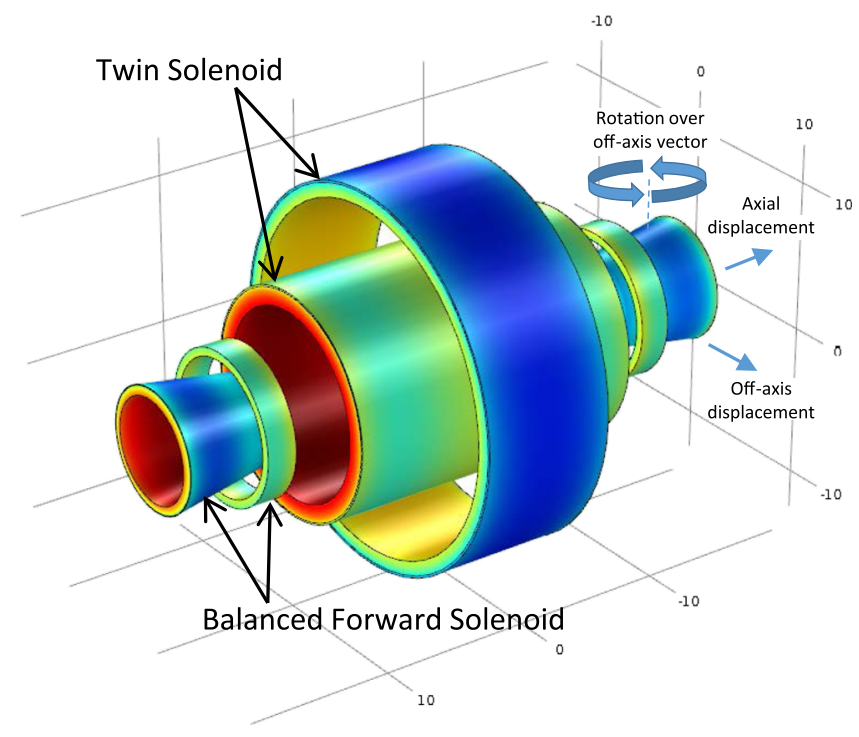

FIG. 4. Three-dimensional representation of the superconducting coils of the twin solenoid and the two balanced forward solenoids. The color indicate the magnetic field on the surfaces of the cold masses, also see Fig. 5. The blue arrows illustrate the various possible misalignments of the cold mass as discussed in Sec. II. 


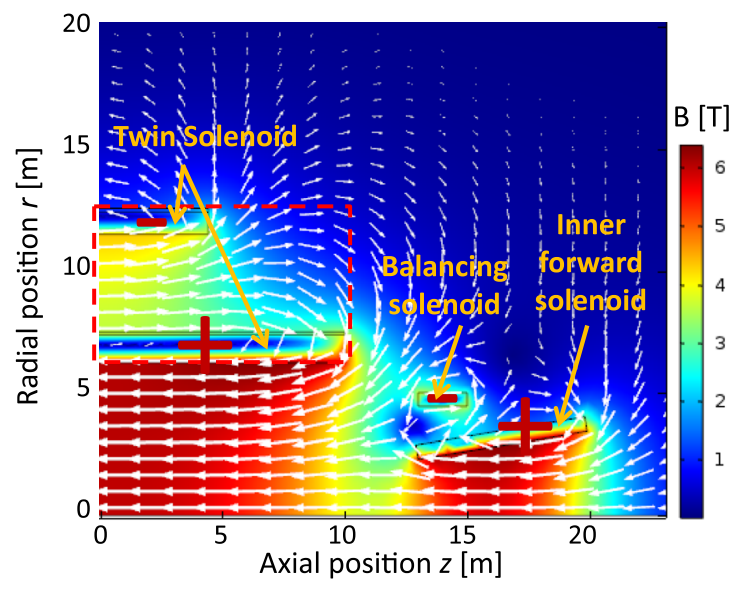

FIG. 5. Two-dimensional axisymmetric representation showing half of the twin solenoid and a balanced forward solenoid. The arrows indicate the magnetic field orientation, while the colors indicate the field magnitude. The plus and minus symbols indicate the direction of current within the solenoids. The red dotted lines indicate the common cold masses.

The balanced forward solenoid is only force and torque neutral if it is in the correct calculated position. With increasing level of misalignment the resulting force and torque increase. If the system is rotated over an off-axis vector or displaced in axial direction (Fig. 4), then the resulting torque and force counters the direction of the displacement. If, however, the cold mass is displaced in an off-axis direction then the net force is directed along the displacement vector. In a detector magnet one may reasonably expect a displacement on the order of several millimeters maximum, which in the case of off-axis displacement would result in a force less than $20 \mathrm{kN}$. If the vacuum vessel of the balanced forward solenoid and the twin solenoid are mechanically connected during operation and relative off-axis movement is constrained by support structure connecting the vacuum vessels to the cold masses, such a force can be handled easily.

\section{ADVANTAGES OF THE BALANCED FORWARD SOLENOID OVER AN EXTENDED TWIN SOLENOID}

The main function of the balanced forward solenoid is to augment the bending power of the twin solenoid for particles with pseudorapidity $|\eta| \geq 2.5$. This may also be achieved by simply extending the length of the twin solenoid. However, this latter option comes with several drawbacks:

The extended twin solenoid requires the same minimum free bore (here: $12 \mathrm{~m}$ ) everywhere to allow for the hadron calorimeter components to be moved in and out of the bore. Thus, whereas the main purpose of extending the magnet system is for tracking high- $\eta$ particles, most of the additional stored energy and thus cold mass would go toward generating a magnetic field in a location which these particles do not traverse. The added cost is not insignificant: to achieve the same magnetic field integral at $|\eta|=4$, the length of the twin solenoid system, the stored energy, and the cold mass would double (Fig. 6).

In addition to the significant extra cost of the magnet itself, there are a number of other reasons why such a design would be unattractive. Being of significantly larger size, the shaft needed to lower the system into the cavern would require a much larger diameter, the crane used for lowering the system would have to accommodate a much higher load, the refrigeration cost would increase, the bore tube holding the tracker and calorimeters inside the magnet system would need significant reinforcement (likely also resulting in a further increase in magnet size to accommodate the larger bore tube), and finally the cavern itself would need to be expanded in order to facilitate the servicing of the tracker and calorimeters.

These considerations should be weighed against the complexity of having additional magnet systems in the forward direction, but overall the use of balanced forward solenoids seems to be beneficial when compared to a significantly extended twin solenoid.

Applying the same argument, the appropriate length of the twin solenoid in combination with the balanced forward solenoids is not given by the performance needed for tracking high- $\eta$ particles, but rather for tracking particles that cannot traverse through the free bore of the balanced forward solenoid (i.e., $|\eta|<2.5$ ). The appropriate balance between the length of the twin solenoid and the free bore diameter of the forward solenoid determines the pseudorapidity-dependent tracking accuracy and is subject for future optimization.

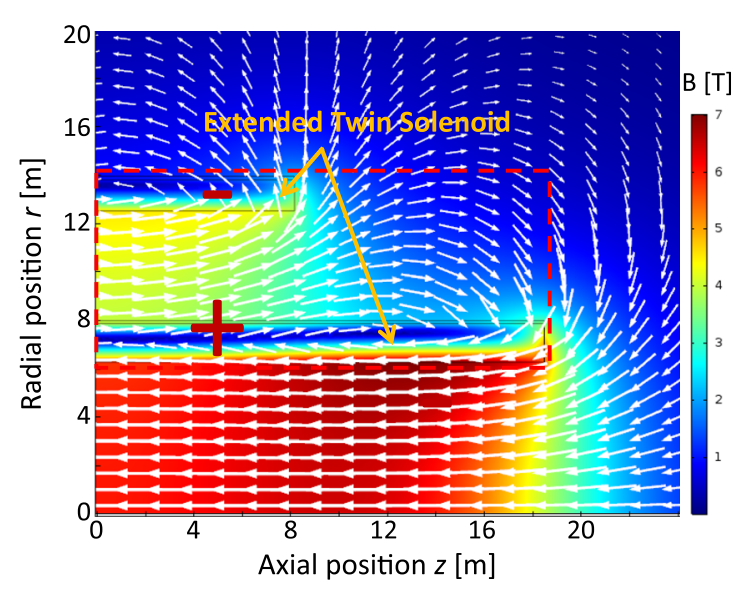

FIG. 6. Two-dimensional axisymmetric representation showing half of a very long twin solenoid. This magnet configuration provides the same field integral at $|\eta|=4$ as the shorter twin solenoid in combination with two balanced forward solenoids (Fig. 5), but the stored energy is more than twice as high. 


\section{ADVANTAGES AND DISADVANTAGES \\ OF THE BALANCED FORWARD SOLENOID VERSUS THE FORWARD DIPOLE}

While choosing the twin solenoid with balanced forward solenoids seems preferable with respect to an extended twin solenoid, the choice between the balanced forward solenoid and a forward dipole is less obvious. In this section, qualitative arguments are given with regard to advantages and disadvantages of either option with a focus on issues concerning the design of the magnet itself, while a quantitative comparison of detector magnet performance is presented in the next section.

First, solenoids are rather inefficient for bending particles traveling nearly parallel to the beam pipe, because the bending power is given by the perpendicular component of the magnetic field with respect to the particle trajectory. In a dipole the magnetic field is oriented perpendicular to the beam pipe which means that it provides nearly optimal bending power for high- $\eta$ particles (Fig. 2).

However, there are several arguments leading to inefficiency in performance per unit of cold mass in dipoles. First, due to the special geometry needed to make the cold mass force and torque neutral without considerably increasing the envelope of the system, about two third of the stored energy in the force-and-torque-neutral forward dipole is a consequence of the magnet field generated by the lateral dipole coils, which do not significantly contribute to the field used for tracking [2]. In comparison, balancing the inner solenoid with the forward balancing coil adds about $12 \%$ to the stored magnetic energy of the balanced forward solenoid. The conductor mass scales with the stored energy, which means that force and torque neutrality comes at a significantly higher cost. Second, due to the lack of radial symmetry, the Lorentz forces inside the conductor are inhomogeneously distributed and the individual coils within the cold mass are not force and torque neutral. Both facts imply that a significant amount of support structure is needed to maintain the mechanical integrity of the system. For instance, in the previously presented preliminary conceptual design two-thirds of the cold mass is support structure [2]. For comparison, in the case of the balanced forward solenoid, the Lorentz forces are radially symmetric so that the reinforced conductor itself can support the forces and each coil is force and torque neutral, so that the amount of support structure is only a small fraction of the total.

The efficiency of bending power per unit volume is determined by the magnetic field magnitude in the bore of the dipole. A key consideration is the ratio of the magnetic field amplitude in the bore with respect to the field on the superconducting conductor itself. For a conduction-cooled magnet utilizing $\mathrm{NbTi}$, which operates at $4.5 \mathrm{~K}$ and should not quench even when exposed to a local temperature increases of some $2 \mathrm{~K}$, the practical upper limit of the magnetic field on the conductor is somewhere below $7 \mathrm{~T}$. In the case of the balanced forward solenoid the current is homogeneously distributed around the free bore so that magnetic the field in the center of the free bore is about $90 \%$ of the maximum field on the conductor. However, the dipole, which has a rather low aspect ratio, is placed in the stray field of the twin solenoid, and needs a significant field in the lateral coils to ensure that the net force and torque on the cold mass is zero, the field in the center is only about a third of the maximum field on the conductor and thus limited to less than $2 \mathrm{~T}$.

From a magnet construction perspective, there is ample experience with regard to building large-scale solenoidal detector magnets whereas no such experience exists for the large aperture dipole. A thorough investigation would be needed to minimize the technological risk associated with the forward dipole, whereas the technological risk associated with the solenoids seems modest.

Yet another argument is related to the trajectory of particles in the area between solenoid and dipole. Since the magnetic field configurations are so different, following particles and keeping them identified is a true challenge for the analysis software.

Finally, the forward dipoles require additional compensation dipoles in order to maintain the alignment of the beam, while the balanced forward solenoids do not have such a requirement.

In summary, as a starting principle utilizing a forward dipole seems attractive, but there are a number of serious disadvantages to a dipole, clearly pointing to the forward solenoid as a preferred option.

\section{COMPARISON OF FIELD INTEGRALS BETWEEN THE BALANCED FORWARD SOLENOID AND THE FORWARD DIPOLE}

In order to make a quantitative comparison of the tracking performance between the balanced forward solenoid and the forward dipole, several assumptions are made as follows.

The balanced forward solenoid and the twin solenoid are assumed to be complementary in terms of field integral, whereas the force-and-torque-neutral dipole is assumed to be a stand-alone system. The latter assumption results from the fact that the interaction between the twin solenoid and the force-and-torque-neutral dipole in terms of particle tracking is complicated. Depending on the azimuthal angle of a high- $\eta$ particle emanating from the interaction point, the bending power exerted by the twin solenoid may complement or partially negate that of the forward dipole. While it may theoretically be possible to utilize the magnetic fields produced by the twin solenoid and the forward dipole in a complementary manner, the preliminary indication of the FCC-hh detector group is that this option is unpractical so that the forward dipole should be considered a stand-alone system. 
To make proper use of the complementary nature of the twin solenoid and forward solenoid, a very precise alignment of the inner and forward trackers is needed. All particles at $|\eta|<2.5$ are assumed to be intercepted by calorimeters located between the interaction point and the magnet systems, while particles at $|\eta| \geq 2.5$ are assumed to traverse through the free bore of the forward magnet system. The outer radial boundary of the inner tracker is at $2.5 \mathrm{~m}$ and the outer axial boundary is at $8 \mathrm{~m}$. The outer boundary of the forward tracker is located at $z=23 \mathrm{~m}$ and this tracker tracks particles at $|\eta| \geq 2.5$.

As a means for comparing the performance of different detector magnet options, the first and second field integrals are used $[2,5]$.

$$
\begin{gathered}
I_{1}=\int_{0}^{L} B_{\perp} d l, \\
I_{2}=\int_{0}^{L} \int_{0}^{L} B_{\perp} d l^{2},
\end{gathered}
$$

where $I_{1}$ and $I_{2}$ are the first and second field integral, respectively, $L$ is the distance between the interaction point and the outermost tracker plane along the path of a given particle, and $B_{\perp}$ is the magnetic field component that is perpendicular to the particle which depending on the orientation of the field with respect to the particle may assume both positive and negative values. Figures 7 and 8 show the first and second field integral of the twin solenoid, the twin solenoid in combination with the balanced forward solenoid, and the forward dipole. In general the first field integral is indicative of the performance for lowmomentum particles, in which multiple scattering plays a significant role, whereas the second field integral is

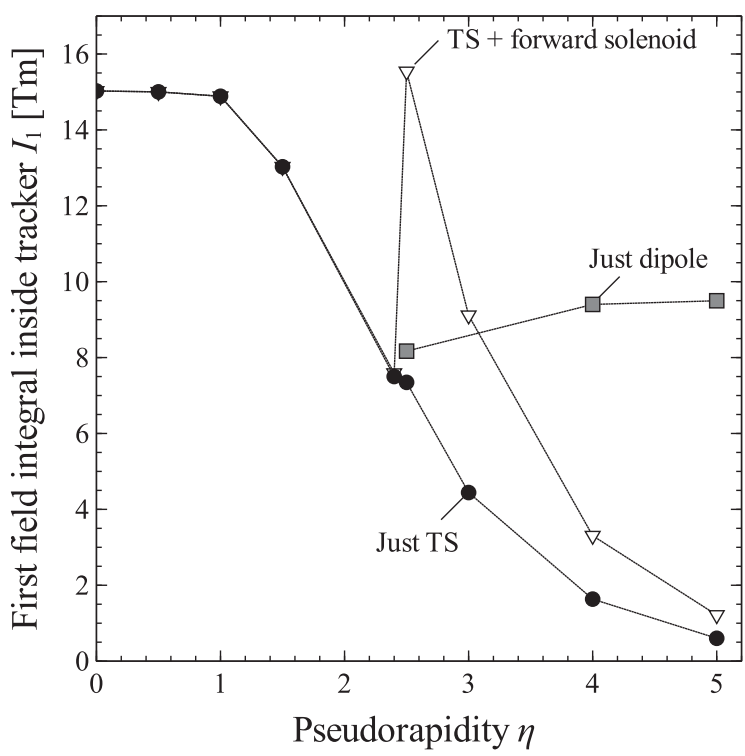

FIG. 7. First field integral $I_{1}$ of the twin solenoid and the balanced forward solenoid versus the forward dipole.

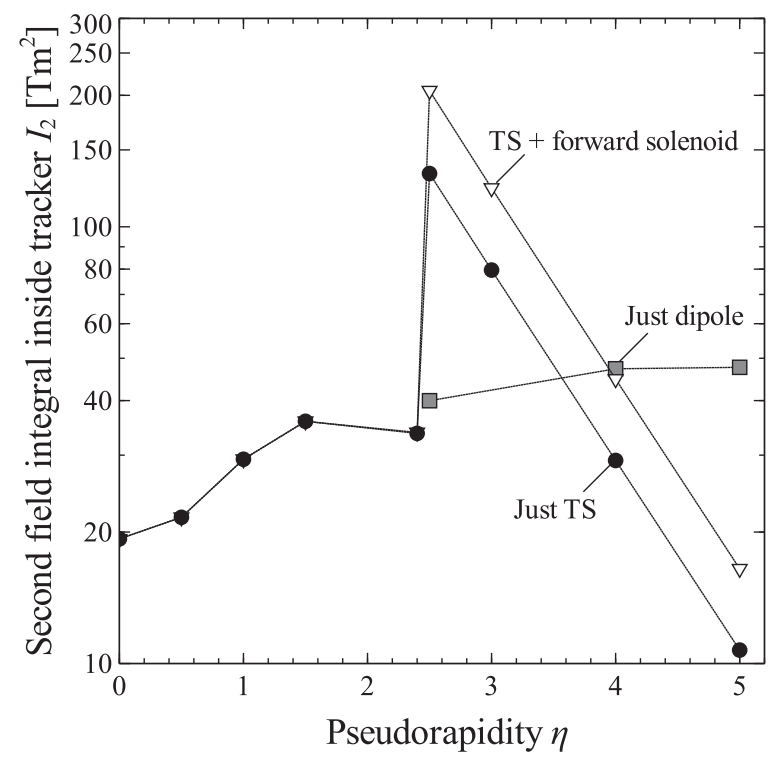

FIG. 8. Second field integral $I_{2}$ of the twin solenoid and the balanced forward solenoid versus the forward dipole.

indicative of the performance of high-momentum particles. A higher field integral indicates better performance.

From these two figures it is clear that the relative performance of the two options of the forward system is dependent on the momentum and pseudorapidity of the particles. For high momentum particles, the twin solenoid with the balanced forward solenoid gives superior performances with respect to the forward dipole in the pseudorapidity regime $2.5 \leq|\eta| \leq 4$ (Fig. 7), whereas for low-momentum particles it is $2.5 \leq|\eta| \leq 3.1$ (Fig. 8). For sufficiently high $|\eta|$, the performance of the forward dipole always exceeds that of the balanced forward solenoid.

A preliminary investigation of momentum resolution as a function of momentum and pseudorapidity supports these general conclusions [6], with the important caveats that this performance relies on very precise alignment of the inner and forward tracker, and that the performance has yet to be investigated in terms of pattern recognition.

\section{DETECTOR MAGNET VARIANTS}

In addition to the twin solenoid with the balanced forward solenoids, two alternative solenoid-based detector magnet geometries were investigated, which are the socalled spherical detector assembly and the unshielded solenoid in combination with balanced forward solenoids. In this section, these designs are discussed in a concise fashion. The stray field and general magnet properties are compared in Secs. VII and VIII.

\section{A. Spherical detector assembly}

The spherical detector assembly, similar to a previously published concept by Green [7], is an attempt to further reduce the stray field and to augment the field integral 


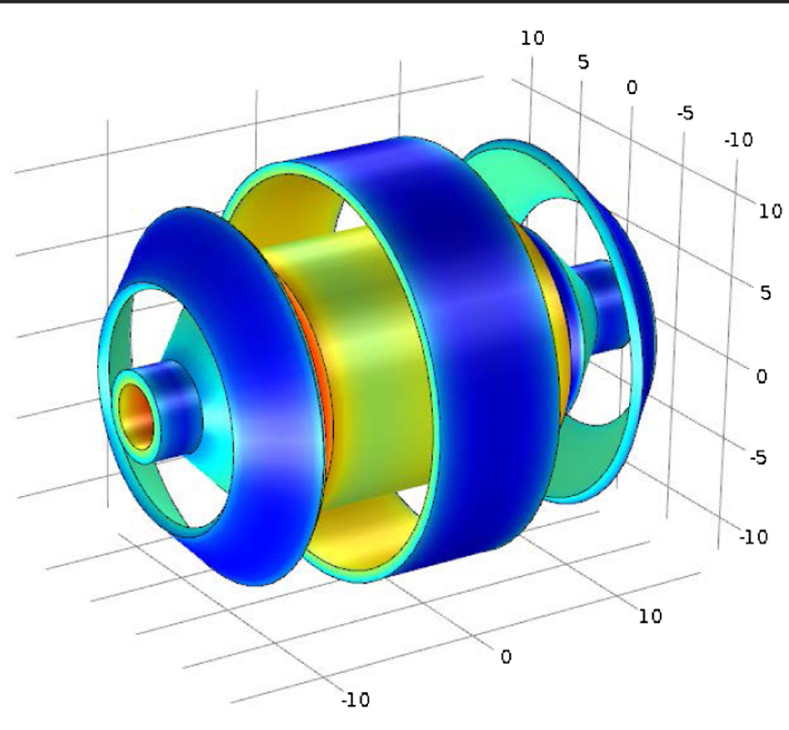

FIG. 9. Three-dimensional representation of the spherical detector assembly. The color indicates the field on the surface of the cold mass (see the legend in Fig. 10).

available for muons in between the inner and outer coils. The name refers to the approximately spherical shape of the overall magnet system (Figs. 9 and 10). The design comprises three shielding coils as opposed to one leading to a significant stray field reduction with respect to the twin solenoid (see Sec. VII, Figs. 13 and 14). For example, in the axial direction the magnetic field drops below $5 \mathrm{mT}$ at about $30 \mathrm{~m}$ away from the interaction point versus about $70 \mathrm{~m}$ for the twin solenoid with the two balanced forward solenoids.

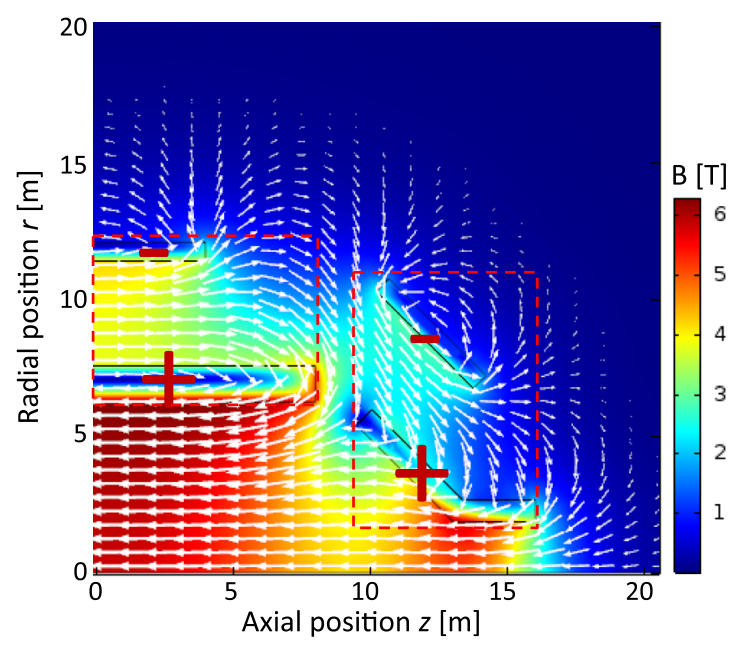

FIG. 10. Two-dimensional axisymmetric representation showing half of the main detector magnet and the forward solenoidal magnet. The arrows and colors indicate the magnetic field orientation and magnitude, respectively. The plus and minus signs in the superconducting coils indicate the direction of the current, while the red dotted lines indicate the common cold masses.
The stray field resulting from this configuration may be manipulated for specific purposes by changing the positions and relative sizes of the coils. For instance, it is possible to optimize the geometry in order to reduce the stray field in front of the detector magnet, so that an ironshielded dipole may be placed there without leading to significant forces or torques. This option is a potential alternative to the otherwise mandatory force-and-torque neutral configuration of the forward dipole. Similarly to the balanced forward solenoid, the two forward solenoidal coils are force and torque neutral so the cold mass is disconnected from the twin solenoid and may be moved during servicing of the calorimeters and trackers. An additional interesting feature is that the magnetic field in between the inner and outer coils is of significant magnitude and aligned approximately perpendicular to the trajectory of particles emanating from the interaction point. This configuration is highly suitable for muon tagging or an independent muon spectrometer system. This design suffers from two major drawbacks. First, while the forward cold mass is torque and force neutral, the individual coils in the forward system are not. Thus cold supports are needed in between these coils to accommodate a tensile force of $650 \mathrm{MN}$. Second, two additional large outer coils are needed to achieve this level of stray field, making this design the most complex of the considered options.

\section{B. Unshielded solenoid with balanced forward solenoids}

The second variant comprises an unshielded solenoid and two balanced forward solenoids (Figs. 11, 12). Due to the absence of a shielding coil, the stray field extends outward much further than with the other considered options, and the available field integral for muon tagging is the least favorable of the three options. For instance, assuming muon chambers are located in the radial range of

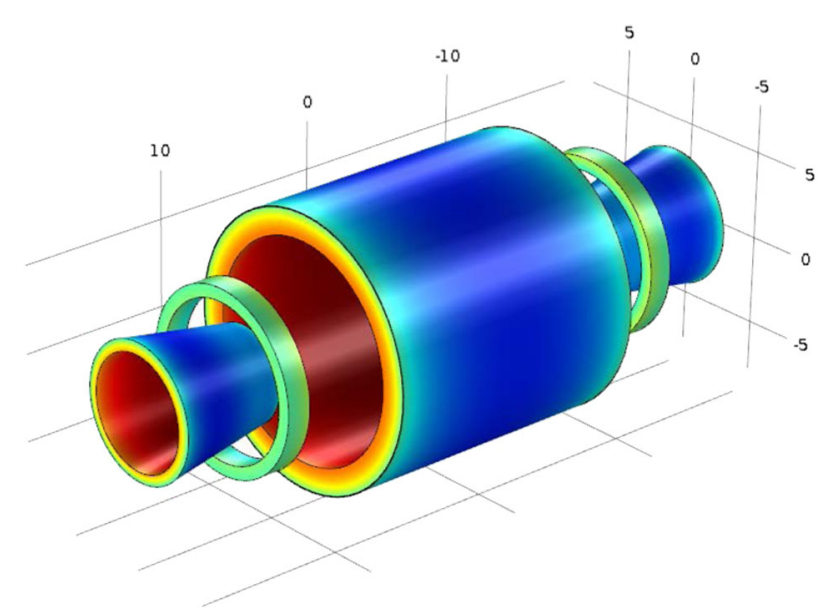

FIG. 11. Three-dimensional representation of the unshielded solenoid in combination with two balanced forward solenoids. The color indicates the field on the surface of the cold mass (see the legend in Fig. 12). 


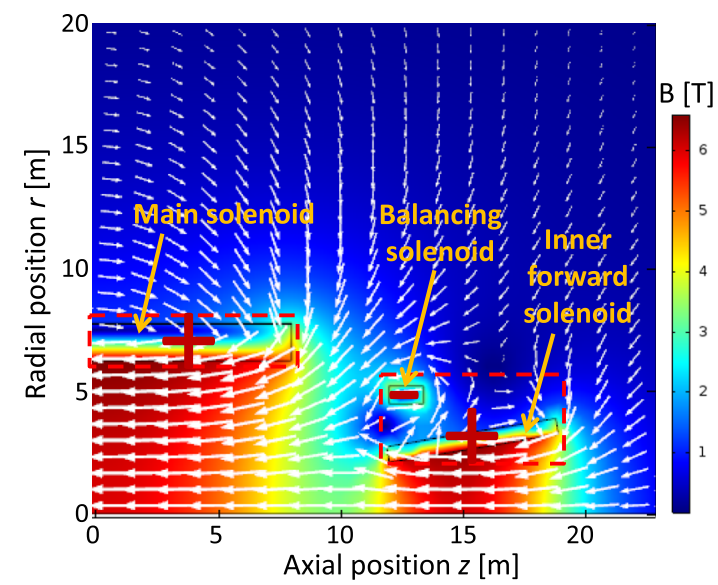

FIG. 12. Two-dimensional axisymmetric representation of half of the unshielded solenoid in combination with a balanced forward solenoid. Here the arrows and colors indicate the magnetic field orientation and magnitude, respectively. The plus and minus signs indicate the direction of the current inside the superconducting coils.

8 to $11 \mathrm{~m}$, the available field integral at $\eta=0$ is equal to $2.6 \mathrm{Tm}$ whereas the space between the inner and outer coils for the twin solenoid gives more than $15 \mathrm{Tm}$. With reliance on muon tagging as opposed to independent muon tracking this lower number may be sufficient, but further study is needed to verify this. To accommodate equipment that cannot operate in a magnetic field, local shielding is required, which could for example mean that the service cavern or substantial parts of it would be surrounded by a layer of iron. The advantage of this variant is that it is the least complex, the lightest, the most compact, and, at least in terms of the detector magnet itself, the most affordable.

\section{STRAY FIELD COMPARISON}

Figures 13 and 14 show the magnetic field magnitude as a function of radial and axial distance to the interaction point. It is clear that the spherical detector assembly option with the three shielding coils has the least extended stray field whereas the unshielded solenoid with the two balanced forward solenoids gives the most. The stray field of the twin solenoid with the two dipoles (not shown here) is about the same as that of the twin solenoid with the two balanced forward solenoids. The stray field of the twin solenoid, as shown here, was specifically optimized to minimize the distance between the interaction point and the $5 \mathrm{mT}$ boundary in the radial direction. Alternatively, it may be optimized to further suppress the magnetic field at larger distance, at the cost of a larger radial distance between the interaction point and the $5 \mathrm{mT}$ boundary and a somewhat increased cold mass.

The detectors are to be placed in caverns some 300-400 meters underground. At such a depth, even the option with the most extended stray field produces a field magnitude at

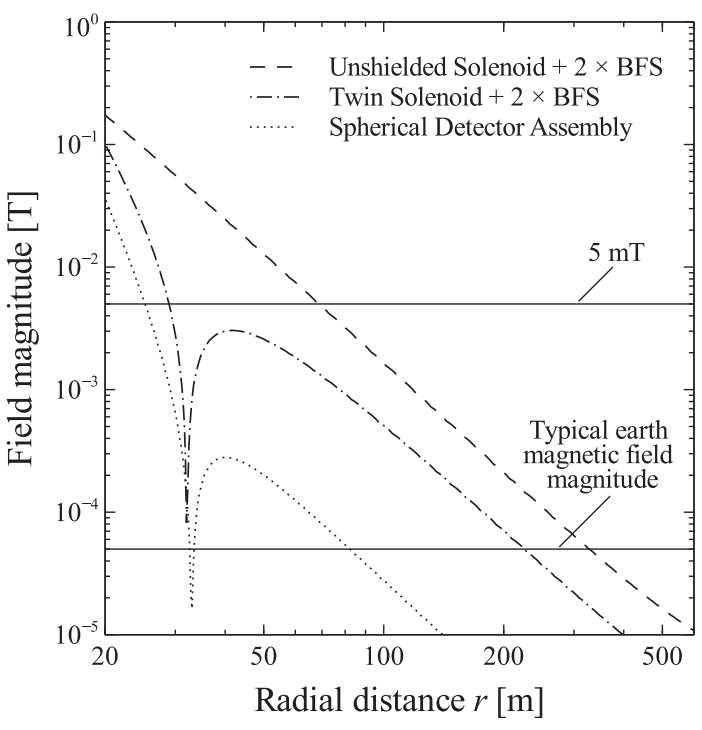

FIG. 13. Magnetic field magnitude as a function of radial distance to the interaction point.

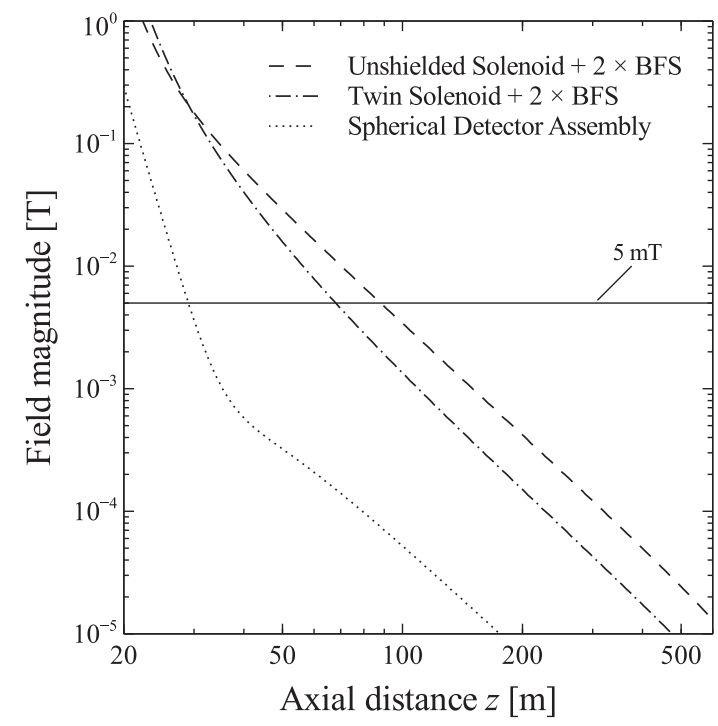

FIG. 14. Magnetic field magnitude as a function of axial distance to the interaction point.

the surface that is at the level of the earth magnetic field. This implies the stray field is unlikely to lead to safetyrelated measures for activities on the surface.

In general it is clear that a reduced stray field, important for operating magnetic-field-sensitive equipment in the vicinity of the detector magnet, comes at the cost of increased complexity. An increase in the amount of shielding coils leads to a reduction in the stray field and vice versa.

\section{GENERAL MAGNET PROPERTIES}

Table I shows an overview comparing main properties of the considered variants. In general, the various magnetic 
TABLE I. Main properties of the magnet variants.

\begin{tabular}{lcccc}
\hline \hline & TS + dip & TS + BFS & SDA & Sol + BFS \\
\hline Cold mass main [kt] & 5.0 & 5.0 & 4.0 & 3.2 \\
Vac. ves. mass main [kt] & 2.5 & 2.5 & 2.2 & 1.4 \\
Cold mass forw. [kt] & 0.3 & 0.5 & 0.8 & 0.5 \\
Vac. ves. forw. [kt] & 0.25 & 0.25 & 0.6 & 0.25 \\
Total cold mass [kt] & 5.6 & 6.0 & 5.6 & 4.2 \\
Total vac. ves. mass [kt] & 3.0 & 3.0 & 3.4 & 1.9 \\
Total stored energy [GJ] & 65 & 68 & 57 & 46 \\
Min. shaft diameter [m] & 28 & 28 & 27 & 16 \\
\hline \hline
\end{tabular}

and mechanical properties are calculated with Comsol multiphysics. Just as in previously published results, advantage is taken of the radial symmetry of the detector magnet variants published here. In the cases where radial symmetry no longer applies, such as when the forward solenoid is displaced in the off-axis direction in Sec. II, Ansys Maxwell is used to perform fully three-dimensional calculations. The two software programs were found to produce consistent results in terms of stored magnetic energy and axial forces. A discussion on uncertainties and limitations of the modeling approach used here is found elsewhere [2].

For all options the field in the center of the main central solenoid is fixed at $6 \mathrm{~T}$, the free bore is fixed at $12 \mathrm{~m}$, the peak Von Mises stress is fixed at just over $100 \mathrm{MPa}$, and the cold masses are assumed to consist primarily out of aluminum [8] with a yield strength that exceeds the $100 \mathrm{MPa}$ stress limit used here. All systems comprising solenoid-based forward magnet systems have a field integral of about $3 \mathrm{Tm}$ at $|\eta|=4$, whereas the twin solenoid with forward dipoles has a field integral of about $10 \mathrm{Tm}$ (also see Figs. 7 and 8).

A rough estimate of the overall vacuum vessel mass is given, which is based on the previously calculated number for the twin solenoid [2]. In Table I the vacuum vessel mass is assumed to scale with the total surface area, with an additional 500 tons for the bore tube of the central solenoid which supports the rather heavy inner calorimeters and the inner tracker. For instance, the vacuum vessel mass of the twin solenoid was previously estimated at about $2.5 \mathrm{kt}$ [2] indicating that the vacuum vessel of each balanced forward solenoid would weigh about 250 tons. The forward dipole, being of similar size, would be about the same as the balanced forward solenoid. The vacuum vessels of the forward solenoids for the spherical detector assembly are much heavier, but this extra weight is compensated by the somewhat smaller vessel size of the central magnet.

With regard to the cold mass of the balanced forward solenoids, a conservative approach is taken where it is assumed that the current density in the forward solenoid is the same as in the main solenoid which results in a cold mass of 500 tons. Then, in the case of a quench the main solenoid and forward solenoid may be discharged in series without leading to a significant temperature rise in the forward solenoid. This is important as it allows for the force and torque free configuration to be maintained during a quench. A more aggressive assumption would be that the current density in the forward system is such that the peak stresses on the conductor in the main and forward system are identical. To balance the rate of temperature increase during a quench between the main and forward systems, the normal state resistivity of the conductor in the main solenoid would then need to be higher than that of the forward solenoid. In this extreme case, the current density in the forward system is $60 \%$ higher than in the main solenoid, and the cold mass would weigh about 300 tons. In reality, the normal state resistivity of the conductor in the central solenoid will be somewhat higher due to the larger fraction of structural material needed to accommodate the Lorentz forces, and the cold mass of the forward magnet system will be somewhere between these two limits.

It is assumed that in the case of the unshielded solenoid the cold mass of the main solenoid can be rotated inside the cavern after being lowered down to the shaft, leading to a comparatively low minimum shaft diameter of about $16 \mathrm{~m}$. If this operation appears not feasible, then the minimum shaft diameter is about $23 \mathrm{~m}$.

In general, the cost advantage of eliminating the shielding coil is clear from Table I, which shows that the unshielded solenoid has the lowest vacuum vessel mass, cold mass, stored energy, and minimum shaft diameter.

\section{DISCUSSION}

In this paper, the case is illustrated that from a magnet construction perspective it is preferable to incorporate a solenoid-based forward system instead of a forward dipole system. From a tracking perspective, either option can be interesting and the implications are presently not fully understood. As such, no specific statement can be made at this time with regard to the relative suitability beyond stating that either option is interesting and that the two options are currently under investigation.

Analogous to previously published calculation results, advantage is taken of the radial symmetry of the detector magnets presented here, so that various properties of these detector magnets may be determined from two-dimensional axisymmetric calculation models. Structural components that do not possess radial symmetry such as for instance the supports between the inner and outer solenoid of the twin solenoid are omitted here. This approach is somewhat simplified but sufficiently detailed to allow for a comparison between different detector concepts. Moreover, the consistent results of a simplified two-dimensional axisymmetric model compared to a more detailed three dimensional model implies that such a two-dimensional approach is reasonable in the initial design phase [2].

The significant cost reduction indicated for the unshielded solenoid should be understood as pertaining 
to the magnet system itself. A careful investigation with regard to the cost of applying local shielding has not yet been undertaken, the cost of which should be compared to the cost reduction achieved by reducing the shaft diameter and the general size and complexity of the magnet system. Important factors here are for example the amount of electronics and rotary equipment to be shielded and the distance to the interaction point. Preliminary estimates indicate that the cost savings achieved by eliminating the shielding coil of the twin solenoid outweigh the added cost of applying local iron-based magnetic shielding to the service cavern by a significant margin.

\section{SUMMARY}

A number of yoke-free solenoid-based detector magnet options for the future circular collider are presented. This includes the new balanced forward solenoid, a system that is placed in line with a main solenoid system for the purpose of enhancing the bending power for low-angle particles. Due to the geometry of this system, the individual coils do not experience a net force and torque during operation, so that a permanent heavy mechanical connection between the cold masses of the main and forward solenoids is not required, thus allowing the forward solenoid to be moved whenever the inner tracker and calorimeters require servicing.

A comparison of the performance of the forward solenoid versus a previously presented forward dipole indicates that the bending power provided by both systems is of comparable magnitude, and further study is needed to determine which of these options is more preferable with regard to other infrastructure and particle physics arguments.

In addition, two other detector magnet configurations are considered where the main difference pertains to the use of shielding coils, and thus the level of stray field and magnetic field integral for muon tracking. This comparison indicates that the considered detector geometries lead to a wide variation in the level of stray field, but that the addition of shielding coils needed for stray field reduction comes at the cost of added complexity, more volume, more mass, increased cooling cost, and an increased minimum shaft diameter.

Towards the conceptual design report of the magnet system for FCC-hh detectors, the various options presented will be further engineered and the conclusions laid down in a the CDR to be issued in late 2018 .

[1] "Future Circular Collider Study", https://fcc.web.cern.ch.

[2] M. Mentink, A. Dudarev, H. Silva, C. Berriaud, G. Rolando, R. Pots, B. Cure, A. Gaddi, V. Klyukhin, H. Gerwig, $\mathrm{U}$. Wagner, and $\mathrm{H}$. ten Kate, Design of a 56 GJ twin solenoid and dipoles detector magnet system for the future circular collider, IEEE Trans. Appl. Supercond. 26, 4003506 (2016).

[3] CMS Collaboration, CMS, the Magnet Project, Technical Design Report, 1997.

[4] V. Klyukhin, A. Hervé, A. Ball, B. Curé, A. Dudarev, A. Gaddi, H. Gerwig, M. Mentink, H. Silva, G. Rolando, H. ten Kate, and C. Berriaud, Superconducting magnet with the minimum steel yoke for the hadron future circular collider detector, J. Supercond. Novel Magn. 3660, 1 (2016).

[5] V. I. Klyukhin, A. Poppleton, and J. Schmitz, ATLAS internal note Report No. INDET-NO-023, 1993, pp. 1-6.

[6] Z. Drasal, Conceptual Study of FCC-hh Tracker Design, in FCC week, Rome (2016).

[7] M. A. Green, A Large Superconducting Detector Magnet Without an Iron Return Path, in Supercollider, Vol. 2 (Plenum Press, New York, 1989), pp. 627-635.

[8] Matweb material property data, Aluminum, Al, www .matweb.com. 